\title{
MAGNETIC SEPARATION IN CZECHOSLOVAKIA
}

\section{VLADIMIR HENCL}

Institute of Geotechnics, V Holesovickach 41, 18209 Prague 8, Czechoslovakia

(Received Janurary 2, 1991)

Abstract The use of magnetic separation in various mineral processing facilities in Czechoslovakia is described. The manufacture of assorted types of magnetic separation machines is highlighted. Potential applications and research and development activities are are discussed.

\section{INTRODUCTION}

The goal of this paper is to present a rather broad review of activities in magnetic separation as it is practiced in Czechoslovakia. Clearly, not all aspects of this technology can be mentioned in this contribution. It can, however, be claimed that all branches of industry in which the application of magnetic separation process is being considered are dealt with.

At the outset it has to be stressed that Czechoslovakia's mineral reserves are limited, with the exception of several ceramic and glass raw material. For this reason, the extent of application of conventional magnetic separation to the beneficiation of typical iron, manganese and chromium minerals, is limited. Recently, a greater attention has been paid to unconventional magnetic separation applications.

For greater clarity this article is divided into three parts: the first part deals with magnetic separation applications to raw materials, the second section includes a survey of magnetic separation equipment manufactured in Czechoslovakia and the third part presents some potential applications including current research activities.

\section{INDUSTRIAL APPLICATIONS OF MAGNETIC SEPARATION}

\section{Iron Ores}

The iron ore reserves in Czechoslovakia are rather small and consist mainly of carbonate-type ores, namely siderites and sidero-plessites, that are only feebly magnetic and can be treated only by high-intensity or high-gradient magnetic separators. 
RudYany

The first application of high-intensity wet magnetic separation in Czechoslovakia, and probably in the world, was the commissioning of the Rudnany plant in 1963.

The Rudnany ore is a complex raw material containing in addition to the main mineral, sidero-plessite $\mathrm{Fe} / \mathrm{MnCa} / \mathrm{CO}$, , also barite, non-ferrous metals sulphides (chalcopyrite, tetrahedrite) and the gangue minerals, such as shale and quartz.

The beneficiation techniques used enable to recover all the useful components; the iron minerals are concentrated by magnetic separation of the ore ground to the particle size of $5 \mathrm{~mm}$, from which the $-0.1 \mathrm{~mm}$ fraction is removed in classifiers.

Originally, 42 units of high-intensity wet induced-roll magnetic separators 2-MSM-5 with the roll width of $1000 \mathrm{~mm}$ were installed for basic separation, together with 42 units of high-intensity drum separators MSB 600 with the drum diameter of $600 \mathrm{~mm}$ and length of $1000 \mathrm{~mm}$, for control separation. All separators are manufactured in Czechoslovakia, each unit having a throughput of $5 \mathrm{t} / \mathrm{h}$ [1].

The separators MBS 600 were later replaced by high-intensity roll separators VMR-1 with double capacity and double length of rolls. Using the feed with the content of 26 to 28 per cent $F e$ the concentrate produced in this circuit contains 36 to 37 per cent $F e$ at 75 to 80 per cent recovery.

Initially, the fine fraction was not treated and was added to the concentrate. In recent years, however, this fine fraction has been upgraded by newly installed high-gradient drum magnetic separators VMS -50 and VMS-100, and by a threorotor high-gradient magnetic separator 6-ERM, produced in Czechoslovakia according to Soviet documentation $[2,3]$ and shown in Fig.1. Similarly, the tailings from the flotation of barite are processed by means of this equipment.

The circuit has a capacity of $60 \mathrm{t} / \mathrm{h}$ and is capable of producing a concentrate of a grade of 36 to 37 per cent Fe from the feed averaging 26 per cent Fe, at the recovery exceeding 80 per cent.

The sidero-plessite concentrate thus produced has the particle size of about 60 to 70 per cent -200 mesh. The concentrate is then dewatered and after adding coke and lime it is micro-pelletized and sintered. The sinter contains more than 50 per cent $F e$ and is further treated in iron plant.

Niźná Slaná

In Nizna Slana plant the ores of metasomatic siderite type [4] are processed at the annual capacity of $750 \mathrm{kt}$. The magnetizing roasting of the ore is employed. 
The grinding of the roasted ore to about 50 per cent -200 mesh is followed by magnetic separation in low-intensity drum magnetic separators MR $9 / 25$ with permanent magnets, of Czechoslovak production.

The basic separation by MR 9/25B separators is followed by two cleaning stages. Magnetic separators MR $9 / 25 \mathrm{C}$ and MR 9/35C are used to clean the magnetic product from the basic separation. The scavenging stage is also included in which magnetic separators MR $9 / 25 B$ are used to treat the middlings from the basic and cleaning stages.

The concentrate of the grade of 51 to 52 per cent Fe at the recovery exceeding 92 per cent is thus produced from the magnetizing-roasted ore, averaging 39 per cent Fe. The concentrate is dewatered and pelletized; the iron pellets are then burned and used in iron plant.

\section{Mẽdënec}

The iron ore from the MYdYnec region [5] belongs to the

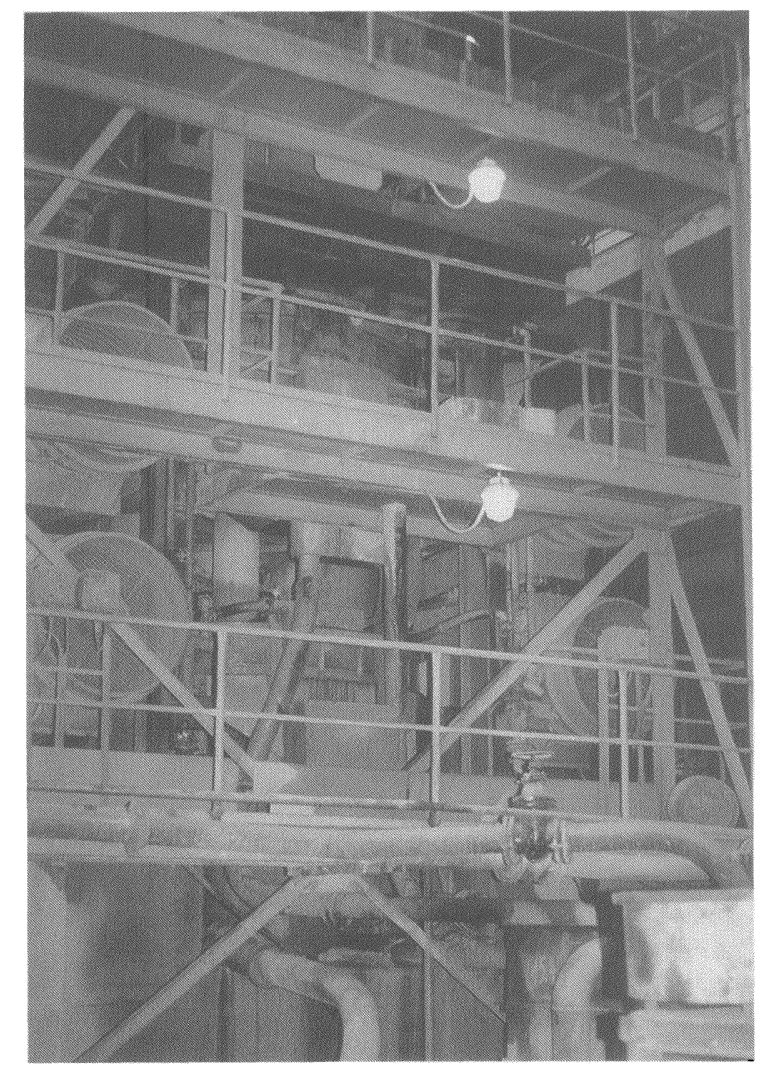

FIGURE 1. The 6-ERM three-rotor high-gradient magnetic separator. 
skarn type; the main components are iron silicates of pyroxene-hedenbergite series, to which 15 per cent Fo is bound. Further components are andradite and magnetite that prevail over garnet, calcite and actinolite. The maximum capacity of the plant is $250 \mathrm{kt} /$ year.

The ore is ground to the particle size of $-25 \mathrm{~mm}$ and then upgraded by a dry drum electromagnetic separator which removes a coarse gangue. The concentrate thus obtained is milled in the first stage to 40 per cent -200 mesh and classified to -200 mesh and +200 mesh size fractions. The +200 mesh fraction is beneficiated in two stages by drum magnetic separator MR $6 / 15$ with permanent magnets, while the -200 mesh fraction is upgraded in three stages. A coarse concentrate from the +200 mesh fraction is milled and beneficiated again in three stages. From the ore with the 36 per cent $\mathrm{Fe}$ content a concentrate of the grade of 67 to 68 per cent $F e$ is produced, and it is used as heavy medium for coal washing.

\section{Magnesite}

The magnesites mined in several deposits in Slovakia, are of braunerite type; that is, they contain an isomorphous mixture of iron. A concentration of $\mathrm{Fe}_{2} \mathrm{O}$, in fired magnesites is 4.5 to 9 per cent. During calcination of these magnesites at temperatures above $800^{\circ} \mathrm{C}$, MgO reacts with $\mathrm{FerO}_{2}$ and magnesium-ferrite $\mathrm{MgO}$. $\mathrm{Fe}_{2} \mathrm{O}_{3}$ is formed.

This ferrite is ferromagnetic and can be separated from the original ore by magnetic separation [6].

In practice, a minimum of 5 per cent of magnesium ferrite must be present in order to obtain, after calcination, a magnetic magnesite clinker. The efficient magnetic separation can be expected only in such a case, when the initial raw material does not contain too much of silicate and dolomite admixtures, because the resulting magnesium ferrite reacts with periclase and $\mathrm{CaO}$ from dolomite and the magnetic properties are then reduced.

It is also necessary to cool down the material slowly after calcination; the growth of magnesium ferrite crystals is thus accelerated.

A proper magnetic separation is not quite easy even when all the optimum conditions for the formation of magnesium ferrite are satisfied. Specific magnetic susceptibility of magnesium ferrite is not higher than $3.8 \times 10^{-5} \mathrm{~m}^{2} / \mathrm{kg}$; it is lower that that of magnetite, and therefore, it is necessary to use magnetic separators with a somewhat increased magnetic field intensity.

The oldest application of this process is the magnetic separation plert in Lubenik that was established in the beginning of this century. At the present time, this tech- 
rology is used also in Hacava plant. The capacity of either plant is about $180 \mathrm{kt} /$ year of clinker.

Various electromagnetic roll separators were used in Czechoslovakia for magnetic separation of calcined magnesite; the most widely used units were Duplex DTU 50120 and Duplex DTUM 501005 machines manufactured by HumboldtWedag (Germany). These separators consist of two rolls with a two-pole magnetic system and have a capacity of $35 \mathrm{~kg} / \mathrm{h}$ per cm of the roll width. The rolls have a diameter of 500 $\mathrm{mm}$ and length of $1200 \mathrm{~mm}$, and the magnetic circuit generates the magnetic field intensity of 145 or $255 \mathrm{kA} / \mathrm{m}$ (1.8 or 3.2 $\mathbf{k O e}$ ).

Some of these electromagnetic separators were successively replaced by dry drum magnetic separators MRVS with permanent magnets. These separators were developed especially for this application in Czechoslovakia.

In the first stage of the technological process of separation the clinker is ground and sized to three size fractions: $-1 \mathrm{~mm},+1-3 \mathrm{~mm}$ and $+3-6 \mathrm{~mm}$. The finest fraction is then processed by separators MRVS $9 / 20$ with permanent magnets (in two stages) and the concentrate, middlings and tailings are thus obtained. The middlings are further beneficiated by high-intensity two-stage magnetic separator WEDAG II $-4-750$.

The +3-6 mm fraction is upgraded by low-intensity drum magnetic separator with bottom feed, magnetic product from which is milled to $-3 \mathrm{~mm}$ and returned back to the circuit.

The +1-3 mm fraction is treated by low-intensity magnetic separators with top feed.

Overall 60 per cent of the concentrate is obtained from the fired raw material. The concentrate thus obtained has the particle size of $-3 \mathrm{~mm}$ and a grade of 90 per cent $\mathrm{MgO}$ and $\mathrm{SiO}_{2}$, up to 3.5 per cent $\mathrm{CaO}$ and up to 6 per cent Fe. $\mathrm{O}_{2}$.

Kaolin

Czechoslovakia is an important producer of first-grade kaolin for both paper-making and chinaware industries and more than 60 per cent of the country's production is exported.

The deposits of the raw material are capable of meeting an increasing demand for high-grade kaolins. However, enormous reserves of raw kaolin are not upgrageable by conventional beneficiation processes.

Such a kaolin is not saleable as a result of contamination by iron and titanium compounds, although other properties are acceptable. Extensive investigation into the properties of these titanium and iron-stained kaolins and into their suitability for beneficiation resulted in a successful 
production-scale solution of the problem.

Kaolin from Kaznejov, which is the largest exploited deposit in Czechoslovakia, is a raw material for the production of kaolin used in paper and rubber industries. Although the floated Kaznejov kaolin is one of the most valued products on the domestic and foreign markets, kaolins of higher whiteness after drying are available abroad.

In Czechoslovakia, a systematic investigation of upgrageability of off-grade kaolins with higher iron and titanium content started in 1955. In 1965 a Jones highgradient magnetic separator was tested. Laboratory results were promising but the production-scale trials did not confirm the laboratory results [7]. A greater success was achieved only in 1979 when SALA-HGMS 107-30-1C high-gradient cyclic magnetic separator with the throughput of $5 \mathrm{t} / \mathrm{h}$ was put in operation.

In 1988 another high-gradient magnetic separator was installed; the EMS 2000 unit manufactured by SKODA Plzen generates the magnetic induction of $2 \mathrm{~T}$ and operates at reduced input power of $0.325 \mathrm{kVA}$ per cubic $\mathrm{dm}$ of separation volume, compared with $0.817 \mathrm{kVA}$ for the SALA separator. The throughput was also increased to $7.3 \mathrm{t} / \mathrm{h}$, thanks to improved construction. By means of HGMS it is possible to increase the kaolin whiteness by 3 to 5 per cent.

\section{THE MANUFACTURE OF MAGNETIC SEPARATORS}

The sole manufacturer of magnetic separation equipment in Czechoslovakia is Bánsko-montány závod (Mining Equipment Plant), a member of Železnorudne bane (Iron Ore Mining Co.) in Spisská Nová Ves [8]. All currently produced separators were developed in cooperation with Ore Research Institute in Mnišek.

The largest percentage of magnetic separators manufactured by the company are low-intensity magnetic separators with permanent magnets (either barium ferrites or strontium ferrites).

Magnetic separators of the MR series are designed for separation of strongly magnetic materials, for regeneration of ferromagnetic heavy media and for separation of undesirable magnetic admixtures from various industrial raw materials.

Drum low-intensity separators are manufactured in various sizes: with drum diameter of 900,1200 and $1500 \mathrm{~mm}$ and length ranging from 600 to $4500 \mathrm{~mm}$. These separators are equipped with three types of a tank: type A - concurrent, type $B$ - counter-current and C-type - semi-counter-current. $A$ special tank designated $R$ and $R A$ is produced for regeneration of heavy media. 
The concurrent and regeneration magnetic separators can treat a material with particle size up to $4 \mathrm{~mm}$, and, depending of the size of a drum, the capacity ranges from 40 to $500 \mathrm{~m}^{2} / \mathrm{h}$.

The counter-current separators can handle material as laree as $2 \mathrm{~mm}$ and their capacity ranges between 100 and $260 \mathrm{t} / \mathrm{h}$. The semi-counter-current magnetic separators can treat a maximum particle size up to $1 \mathrm{~mm}$ and have a capacity of 20 to $350 \mathrm{t} / \mathrm{h}$.

Magnetic separators of the MRS and MRVS series are suitable for dry separation of strongly magnetic materials and for separation of undesirable ferromagnetic admixtures from various industrial raw materials. These drum separators are available with drum diameter of $900 \mathrm{~mm}$ and $1000 \mathrm{~mm}$ and length of $2000 \mathrm{~mm}$ (MRS), or with drum diameter of $600 \mathrm{~mm}$ (MRVS).

The throughput of these separators ranges from 5 to $20 t / h$ at maximum particle size of $20 \mathrm{~mm}$ (MRS) or from 2 to 5 t/h maximum particle size of $6 \mathrm{~mm}$ (MRVS). The separator MRVS is especially constructed for separation of magnesite clinker.

Magnetic separator MRO was developed for regeneration of lubricating and cooling emulsions. It is also equipped with permanent magnets and is used for separation of iron scale, generated during rolling and grinding operations.

The drum of the separator has a diameter 600 or $900 \mathrm{~mm}$, and the length from 660 to $1500 \mathrm{~mm}$. Maximum particle size is 5 $\mathrm{mm}$. Magnetic separators MRO are capable of regenerating 30 to $90 \mathrm{~m}^{\text {* }}$ of emulsion per hour.

Drum magnetic separators of MBO series are manufactured for the removal of iron objects from loose material transported on conveyor belts. Separators MBO are manufactured with a diameter ranging from 315 to $1000 \mathrm{~mm}$ and are suitable for conveyor belts 400 to $1200 \mathrm{~mm}$ wide.

In addition to separators MBO, the magnetic belt separators MPO are used for continuous removal of undesirable iron objects transported on the belts. Separators MPO are either suspended or self-supporting, are usually placed above a continuous conveyor and are mounted perpendicularly to the direction of conveyor axis.

The separators are equipped either with an electromotor with power input of $2.2 \mathrm{kw}$, or with a magnetic circuit that consists of permanent magnets, for the belt width of up to 800 mm.

For magnetic separation of weakly magnetic materials, magnetic separators MSM are manufactured. These separators employ an electromagnetic system comprising pole-pieces and 
two induction rolls. These rolls have a diameter of $270 \mathrm{~mm}$ and length of $1000 \mathrm{~mm}$. Throughput of the separator is 3 to 6 $t / h$ at maximum particle size of $5 \mathrm{~mm}$. In a modified version. the particle size up to $10 \mathrm{~mm}$ is admissible. The MSM magnetic separator is shown in Fig. 2.

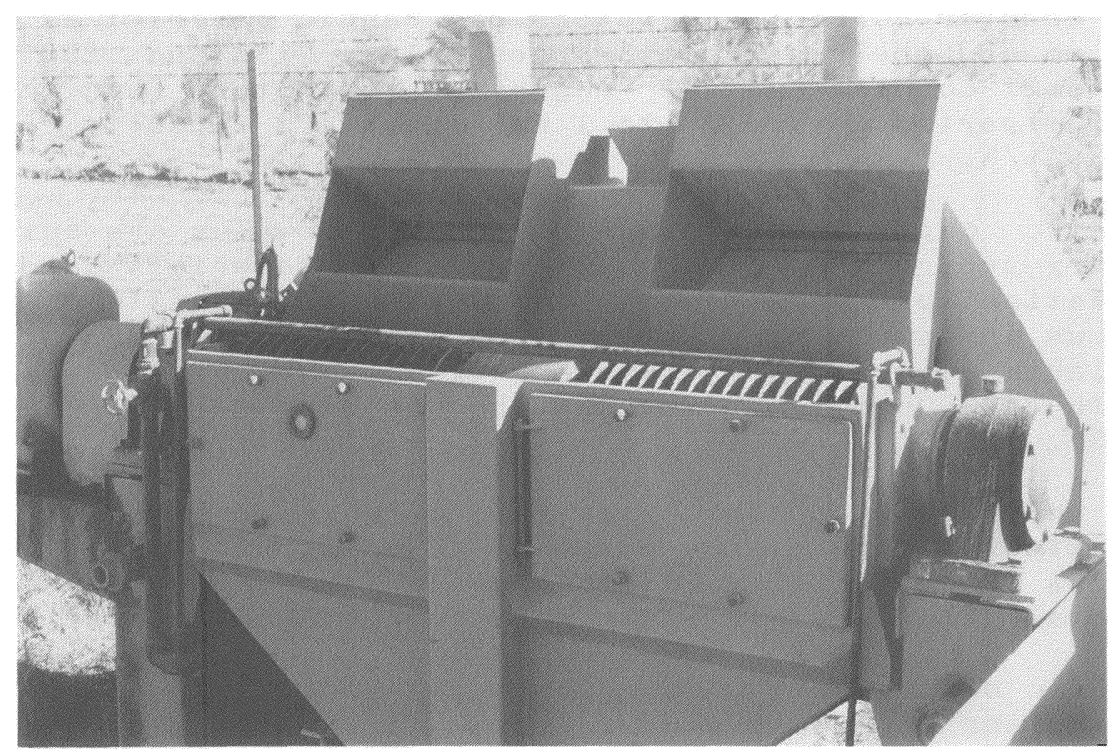

FIGURE 2. The MSM high-intensity magnetic separator.

For separation of paramagnetic, very fine raw materials a high-gradient magnetic separator VMS is manufactured. The separator is of modular design, each separation unit having one rotor with diameter of $2000 \mathrm{~mm}$ and width of 500 or 1000 $\mathrm{mm}$.

According to the number of modular units, the separator has one or two rotors. The machines generating the magnetic field of $0.5,1.0$ and $1.5 \mathrm{~T}$ have been manufactured. The VMS separators are capable of treating 25 to $120 \mathrm{t} / \mathrm{h}$ of material with the particle size of 0.01 to $1 \mathrm{~mm}$. The matrix is made of $3 \mathrm{~mm}$ diameter rods, the distance between the rods being 1.5 or $2.5 \mathrm{~mm}$.

The optimum regime of the separation process can be simply set and can be monitored on a control panel. A complete VMS circuit also comprises control sieves and low-intensity magnetic separator MR. These auxiliary units control the upper limit of the particle size and the removal of strongly magnetic admixtures from the feed.

\section{RESEARCH AND DEVELOPMENT}

Within the framework of further development, design of a 
continuous high-gradient magnetic separator for kaolin beneficiation was undertaken. The machine was successfully commissioned and it eliminates the deficiencies of cyclic separators that are currently in operation world-wide.

Continuous separators do not require periodical switching on and of $f$ of the magnetic field, necessary for rinsing and flushing the loaded matrix. These separators thus have larger duty cycle and higher throughput. Continuous separators can also eliminate progressive deterioration of quality of treated kaolin which occurs at loading stage in a cyclic separator.

In the first phase of the development, carried out by the Ore Research Institute, a pilot-plant continuous separator VMKS-1 was built. The separator has a capacity of $1 t / h$ [9], matrix surface of $0.27 \mathrm{~m}^{2}$, magnetic induction of $2 T$ and the flow velocity of the slurry can be controlled up to 20 $\mathrm{mm} / \mathrm{s}$. The separator has dimensions $4.45 \times 2.62 \times 3.88$, woighs $34.5 t$ and the input power amounts to $170 \mathrm{~kW}$ (at magnetic induction of $1.5 \mathrm{~T}$ ) and $400 \mathrm{~kW}$ (at magnetic induction of 2 T).

When tested on kaolin suspension from Sadov plant, it was possible to decrease the $\mathrm{Fe}_{2} \mathrm{O}_{3}$ and $\mathrm{TiO}_{2}$ content from the head value of 1.36 per cent to 0.98 per cent and to increase the whiteness by 3 to 5 per cent.

On the basis of these successful results the productionscale magnetic separator VMKS-15 was constructed. The separator has a capacity of $10 \mathrm{t} / \mathrm{h}$ and matrix surface 2.5 $\mathrm{m}^{2}$. The separator has dimensions $6.2 \times 5.1 \times 5.7 \mathrm{~m}$, the rotor diameter $3000 \mathrm{~mm}$ and width $1380 \mathrm{~mm}$. The machine weighs $260 \mathrm{t}$ and the power input at the maximum magnetic induction of $2 \mathrm{~T}$ is $320 \mathrm{~kW}$.

Schematic diagram of the separator is shown in Figure 3 . The magnetic system consists of the coils of rectangular crosssection (1), surrounded by iron return frame (2). The lower part of the iron return frame is perforated (3) to allow passage of the slurry; the upper part (4) is slotted (5) for the magnetic fraction to be removed.

A cylindrical trough with inlets (6) is placed in the magnetic system. The trough is connected to two pipes (7) for the magnetic fraction removal. The rotor (8) having the matrix (11) placed in compartments (10) along the rotor circumference, is inserted into the trough. The rotor that employs stiffeners (9) rotates on a shaft (13) and is fitted with seals on both side walls which prevent the suspension from by-passing the matrix.

The matrix is fixed in both lower and upper parts of the compartments by caps (12). A flush station (15) is installed above the upper part of the rotor, while the magnetic fraction is collected inside the rotor (16). Production-scale 


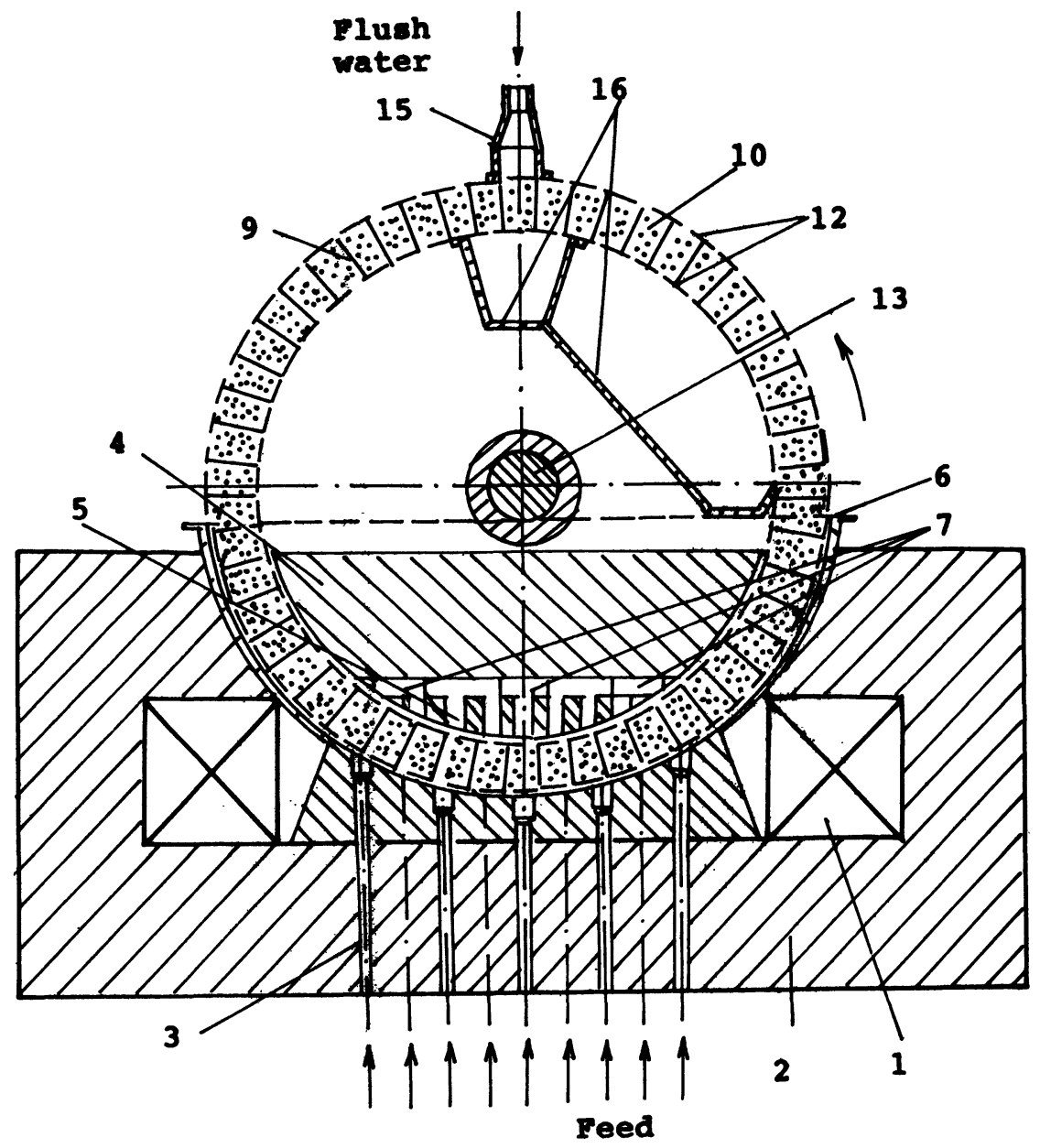

FIGURE 3. Schematic diagram of high-gradient magnetic separator VMKS-15.

trials of the separator will commence in 1991. The overall view of the VMKS separator is shown in Fig. 4.

In recent years an attention was paid to the application of magnetic separation, namely of magnetic filtration in the cleaning of industrial and drinking waters.

The fundamental research was carried out at the Institute of Geotechnics of the Czechoslovak Academy of Sciences in Prague. The application of HGMS is especially suitable for the water which contains magnetic impurities (e.g. steel plant waste water) and presently numerous magnetic filters are in operation all over the world.

Magnetic filters operating in a cyclic mode are disadvantageous, in view of a rather short duty cycle, particularly 


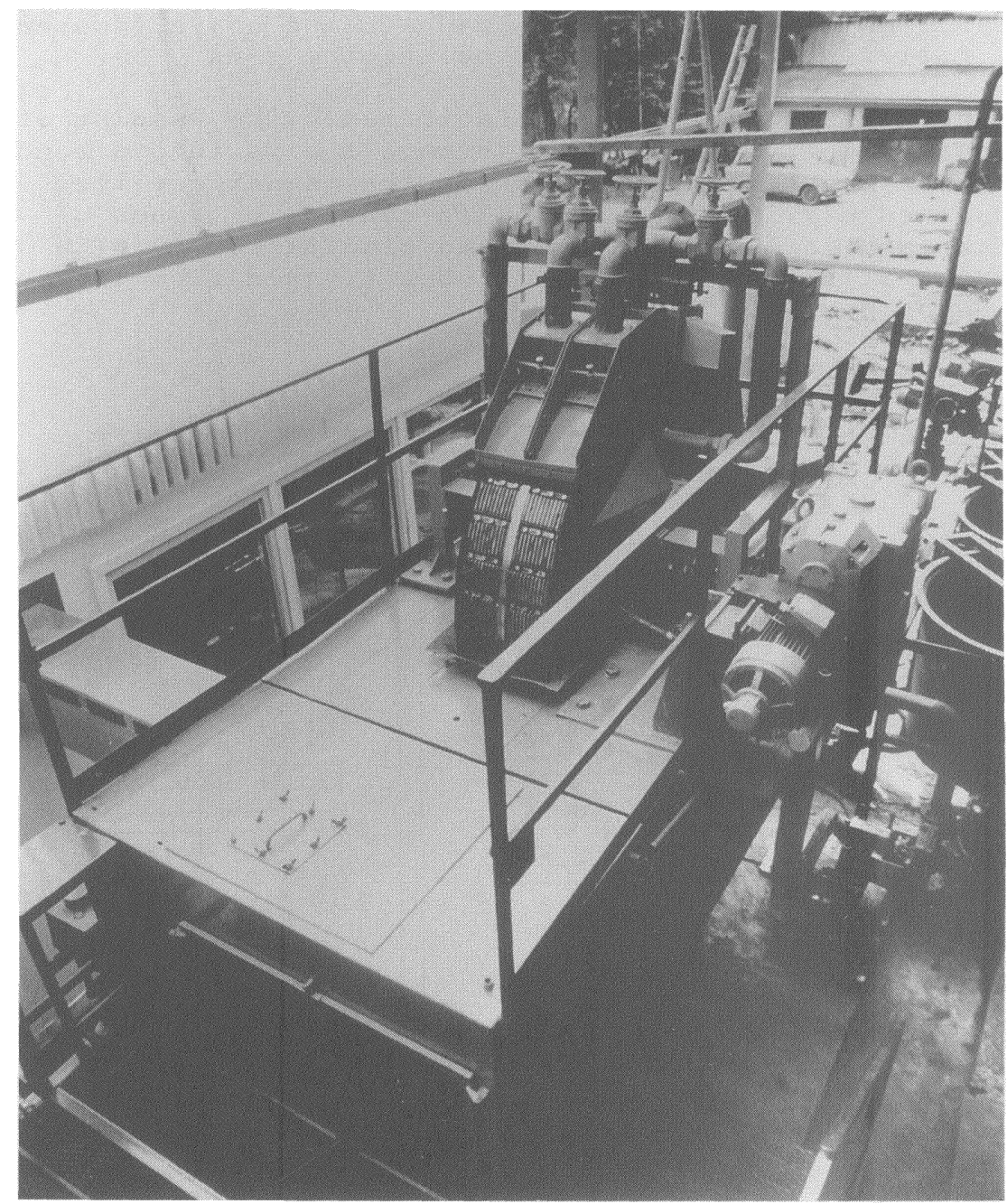

FIGURE 4. High-gradient magnetic separator VMKS-15.

when concentration of magnetic impurities in the waste water is not small, which is usually the case. In such applications the utilization of the machine is very low and viability of such a process is limited, in view of a high cost of such a machine.

To improve this situation, a model of a new magnetic filter which operates in a combined regime was developed in Czechoslovakia [10].

Schematic diagram of the circuit for filtration in a magnetic field is shown in Figure 5 . The circuit consists of a tank (1), a pump (2), the outlet of which is fitted with a bypass whose capacity can be controlled by valves $(3,4)$. The inlet pipe is equipped with a back-flow flap (5) which 
prevents the intake of pressurized air and of a suspension to be filtered back into the pipe and the tank.

The pressurized air which facilitates the washing of the filter is fed from an air-bag through a pipe into a bend in front of the entrance chamber of the magnetic filter. The pressurized air is under hydraulic control by means of valve C which is being closed and opened simultaneously with outlet valve $B$ through which the sludge flushed from the matrix $M$ is removed.

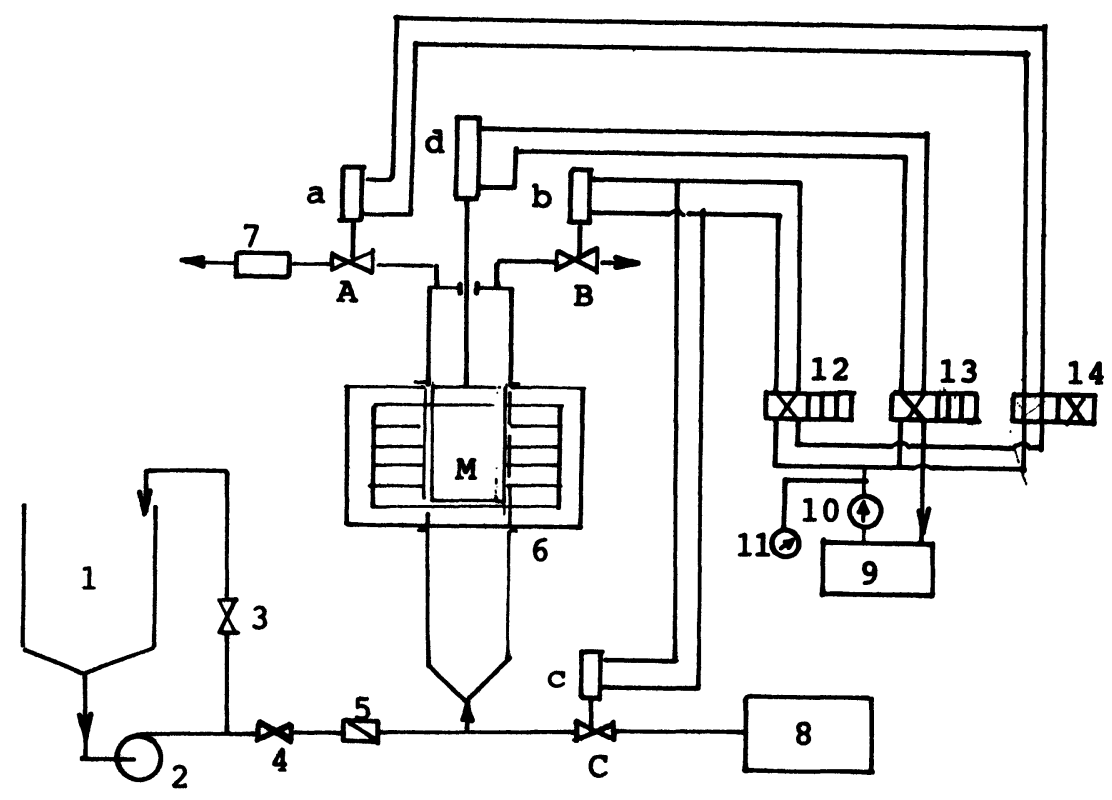

FIGURE 5. Schematic diagram of magnetic filtration circuit.

Hydraulic energy is supplied by internal gear-pump [10] which operates in an open hydraulic circuit. Electrohydraulic distributors $(12,14)$ control the opening and closure of valves $A, B$ and $C$ (valves $B$ and $C$ open and close simultaneously). The electrohydraulic distributor (13) controls the lifting and replacement of the matrix-filled canister.

The hydraulic circuit is driven by linear engines a, b, c and d. The flow of hydraulic fluid in the outlet branch of hydrogenerator is regulated by an externally controlled bypass valve so that during an interval when the pistons of hydroengines are not in motion the hydraulic fluid passes directly into the tank (9), its pressure being determined 
only by flow resistances.

The pressure in the hydraulic circuit is indicated by manometer (11). Velocity of piston motion can be controlled by one-way throttle valves. Flowmeter (7) is placed in the purified water outlet pipe after valve $A$.

All functions of the magnetic filter can be controlled manually or automatically. In automatic control mode the required filtration time is adjusted by a time-switch. After a pre-set time interval has elapsed, the time-switch closes the valve of the purified water. The closure of the valve is indicated by an end-switch which initiates the lifting of the matrix from the magnetic field.

As soon as the matrix has reached its upper position, the valve $B$ (sludge removal) is opened simultaneously with valve $C$ (pressurized air). During the movement of the matrix, the velocity of which can be regulated, into the initial position the sludge captured by the matrix is washed of $f$.

When the matrix has reached its initial position (in the magnetic field), valve $B$ is closed while the valve $A$ is simultaneously opened and a new filtration cycle begins.

During the lifting of the matrix from the magnetic field the valves $A$ and $B$ are closed (valve overlap). During a short interval when the closure of two valves overlaps, the bypass to the tank (1) increases for a moment.

The functional model has the following specifications: magnetic field can be adjusted up to $0.7 \mathrm{~T}$, the throughput is 3 to $51 / \mathrm{s}$ and the duty cycle is as high as 97 per cent.

The water containing non-magnetic impurities can usually be treated by magnetic filtration by adsorbing the impurities onto a magnetic carrier, often finely ground magnetite. Preparation of of sufficiently fine fractions of magnetite is rather demanding and therefore costly. Moreover, such fine particles do not have sufficiently high surface. As a result, relatively high carrier additions are necessary. It follows, for instance, from tests performed in Australia [11] that to purify drinking water by magnetic seeding, 12 g/l of magnetite with particle size between 1 and 5 micrometers is required, at the cost of AUS\$ 100 per ton.

For this reason, the application of chemically precipitated magnetite from waste solutions of $\mathrm{FeSO}_{4} .7 \mathrm{H}_{2} \mathrm{O}$ has been developed at the Institute of Geotechnics. Procedure described by Okuda [12] has been employed: magnetite of particle size of 1 micrometer can be used at somewhat reduced concentration. The regeneration of magnetite is then possible by adjusting the surface charge by changing $\mathrm{pH}$ and by applying HGMS.

It has been proposed to use, for this purpose. the modified 
HGMS separator VMKS, in which lower magnetic induction of $0.5 \mathrm{~T}$ is generated. The mass and the price of the separator is thus considerably reduced and, at the same time, high throughput is maintained because of the continuous mode of operation.

\section{REFERENCES}

1. V. Hencl, in Proc. VIth International Mineral Processing Congress, Cannes, France, 587 (1963)

2. J. Cibulka et al., in Proc. XVth International Mineral Processing Congress, Cannes, France, 363 (1985)

3. L. Bartos et al., in Proc. Conf. "Theoretical and practical problems of magnetic separation of mineral raw materials". Strbske Pleso, Czechoslovakia, 51 (1989)

4. V. Cisko, in Proc. Conf. "Theoretical and practical problems of magnetic separation of mineral raw materials", Strbske Pleso, Czechoslovakia, 111 (1989)

5. V. Hencl, Freiberger Forschungshefte A 231 , Apv. 45 (1962)

6. V. Hencl and O. Kolar, Verfahrentechnik, 11, 503 (1967)

7. J. Baburek et al., in Proc. Conf. "The application of superconductivity in magnetic separation", Prague, 69 (1979)

8. Anon. Brochures published by Iron-ore Minins Co.. Spisska Nova Ves

9. V. Zezulka and F. Zurek, in Proc. XVIth International Mineral Processing Congress, Stockholm, Sweden, 1075 (1988)

10. V. Hencl and P. Mucha, in Proc. XVIth International Mineral Processing Congress, Stockholm, Sweden, 351 (1988)

11. N.J. Anderson and A.J. Prestley, Water Research, 17, 1227 (1983)

12. T. Okuda et al., Filtration and Separation, 9,442 (1975)

V. Hencl received his M.Sc. degree in chemistry from the Charles University in Prague, Czechoslovakia, in 1952. In 1974 he was awarded the D.Sc. degree by the Technical University in Kosice, Czechoslovakia. 
His professional carrier began in the Ore Research Institute in Prague in 1952. He was involved mainly in the development of magnetic separation process. In 1978 he joined the Institute of Geotechnics of the Czechoslovak Academy of Sciences in Prague as a chief scientist and Head of department. He is currently involved in the development of new technologies, mainly for waste and waste water treatment.

Keywords: magnetic separation, iron ores, magnesite, kaolin, manufacturers of magnetic separators, Czechoslovakia. 